\title{
Desarrollos recientes en las reformas administrativas en el sureste asiático
}

\section{Mohammad Mohabbat Khan *}

\section{Introducción}

Cada vez hay más preocupación porque las rígidas estructuras de gobierno son inadecuadas e inapropiadas en un mundo constantemente cambiante, complejo e interdependiente. Existe una clara necesidad de llevar a cabo reformas amplias y de gran calado. El objetivo es conseguir instaurar la eficiencia, la eficacia y la economía en las operaciones y actividades de la función pública.

Este artículo es un intento de describir y analizar reformas administrativas recientes en países seleccionados del sureste asiático. Los países elegidos para este examen son Hong Kong, Malasia y Singapur. En la región estos países han hecho avances significativos en el terreno de la reforma administrativa. La exposición que sigue se estructura de manera lógica. Se ofrece una descripción breve sobre los tres países. También se revisan desarrollos recientes en torno a la reforma administrativa. La parte más importante del artículo se concentra en recientes reformas administrativas significativas en Hong Kong, Malasia y Singapur. En la última sección el análisis se centra en los factores y variables que contribuyeron a la implementación con éxito de medidas significativas de reforma administrativa y las lecciones que se desprenden de estos éxitos para otros países.

Hong Kong cubre un área de 1.044 kilómetros cuadrados incluyendo dentro de él 235 islas y es una parte de una península que sobresale hacia el sur desde China meridional (HuQue, 1996:4). La abrumadora mayoria, es decir, el 98\% de los 6 millones de habitantes es china y hablan en cantonés. El PNB per ca- pita es de 10.350 \$USA (Banco Mundial, 1991). Hong Kong fue una colonia británica desde 1842 hasta mediados de 1997. Desde el 1 de julio de 1997 Hong Kong está bajo soberanía china. Tiene el status de una región administrativa especial dentro de China. El sistema político de Hong Kong se caracteriza por un Ejecutivo Principal, nombrado por las autoridades políticas en Pekín, un Consejo Legislativo y una rama ejecutiva (Lo, 1998).

Malasia cubre un área de 329.758 kilómetros cuadrados, teniendo como vecinos inmediatos a Brunei, Singapur, Tailandia e Indonesia (AHMAD, 1998). La población actual es de 21 millones de habitantes. Más del 50\% de la población es malaya seguida de los chinos con el 33\% y los indúes que son el 10\% (QuaH, 1990:238). La renta per capita alcanzó los 4.027 \$USA en 1995 (AнmaD, 1998). Malasia ganó la independencia de los británicos en 1957 como Malaya. En 1963 se convirtió en Malasia con la unión de los dos Estados de Borneo, de Sarawak y Sabah. Malasia tiene un sistema parlamentario con una monarquía constitucional en la cúspide. Los idiomas más importantes son malayo, chino (mandarín) y tamil.

Singapur es una ciudad-Estado con un pequeño territorio de 224 millas cuadradas que tiene una población estimada de 3.004.300 habitantes (SINGAPORE, 1997a: 4). De la población total, existe una abrumadora mayoría de chinos, es decir, el $77,3 \%$ seguidos por malayos 14\% e indúes 3,3\% (SINGAPORE, 1997a: 4). Las cuatro lenguas oficiales son el malayo, el chino (mandarín), tamil e inglés. Entre éstos, el malayo es el idioma nacional, mientras que el inglés es el lenguaje oficial. La renta per capita es de 25.000 \$USA y el PNB es de más de $39.000 \$$ USA (SINGAPORE, 1997a: 39). Singapur fue una colonia británica y se convirtió en independiente en 1959. 


\section{Desarrollos anteriores en la reforma administrativa}

Los desarrollos en el terreno de la reforma administrativa en los tres países no han sido parejos aunque se pueden destacar algunas similitudes. En Hong Kong la reforma administrativa no fue un área prioritaria antes de 1989. Hubo intentos de lo que se pueden llamar esfuerzos de modernización administrativa para mantener el statı quo. El gobiemo se reorganizó bajo la administración Maclehose sobre la base de recomendaciones contenidas en el informe Mckinsey de 1973 (LO y YUEN, 1994: 3). Uno de los resultados de las recomendaciones Mckinsey fue la creación de sectores políticos y de recursos dentro del gobierno. La planificación a largo plazo, como resultado de la reforma, se convirtió en una responsabilidad crucial del gobierno. Comenzó la regionalización. Se diseñaron setenta y cinco planes programáticos en áreas tan importantes como los servicios médicos y de salud, vivienda, bienestar social y educación.

Existía una corrupción generalizada en la función pública de Hong Kong. Pero la situación cambió con el establecimiento de una Comisión Independiente contra la Corrupción en 1974. A esta Comisión se le confirió una amplia gama de poderes. Tenía el poder de arrestar a la gente que estaba bajo sospechas, buscar y retener sin una orden de arresto, requerir información, congelar los bienes y la propiedad e impedir que la gente abandonase el país (CAIDEN, 1991: 249). La Comisión estaba asistida en esta operación por cinco comités consultivos sobre la corupción, revisión de operaciones, prevención, relaciones comunitarias y reclamaciones, que estaban formados por sectores representativos de la población y que informaban directamente al gobernador (CAIDEN, 1991: 249). Se organizó en tres áreas para llevar a cabo de manera efectiva sus deberes y responsabilidades. Las áreas eran: a) un departamento de operaciones para investigar, arrestar y ayudar en el procesamiento; b) un departamento de prevención de la corrupción para restructurar las organizaciones gobernamentales de forma que se reduzcan las oportunidades para la corrupción y c) un departamento de relaciones comunitarias para cambiar las actitudes de las personas hacia la corrupción (CAIDEN, 1991: 249).

En Malasia la reforma administrativa fue un objetivo importante de la política del gobierno desde los mismos comienzos. Esto era así porque el gobierno encargó las tareas de desarrollo socioeconómico y construcción de la nación a la función pública. En los años 60 las reformas más importantes incluyeron el establecimiento de la Unidad de Desarrollo Administrativo dentro del Departamento del Primer Ministro, la introducción del Sistema de Presupuesto de Programa y Funcionamiento (AHMAD, 1998). La Unidad de Desarrollo Administrativo se responsabili- zaba de identificar e implementar mejoras administrativas en la función pública. En los años 70 se introdujeron una serie de medidas de reforma. Estas medidas incluían: el establecimiento de un Departamento de Servicio Público independiente, así como la Unidad de Planificación de Personal y de Modernización Administrativa de Malasia, conocida ahora como la Unidad de Planificación de Gestión y de Modernización Administrativa de Malasia, en 1977, y el Instituto Nacional de Administración Pública, en 1972 (AHMAD, 1998). El Departamento de Servicio Público trataba de mejorar la capacidad y efectividad de todas las organiza. ciones del sector público por medio de la iniciación y ejecución de políticas de gestión de personal. La Unidad de Planificación de Gestión y de Modernización Administrativa intentaba estudiar e identificar problemas administrativos importantes en todos los niveles del gobierno y recomendar cambios necesarios y medidas innovadoras apropiadas para superarlos. El Instituto Nacional de Administración Pública se fundó con el objetivo de incrementar la capacidad y efectividad en todas las agencias públicas para formular e instrumentar políicas y programas de desamollo a través de la formación (RAHMAN, n.d.). Subsiguientemente se estableció una División de Desarrollo de Carreras y de Formación dependiente del Departamento de Servicio Público y el Programa de Excelencia en la Función Pública se inició en 1979.

Singapur, al igual que Malasia, inició las reformas administrativas después de la independencia. Las reformas iniciales tenían el propósito de modelar las actitudes de los funcionarios públicos y conseguir instaurar algunos cambios estructurales en la función pública (KHAN, 1998: 383). Las reformas se concentraron en la reorganización estructural de la función pública de Singapur mediante la creación de nuevos ministerios y la racionalización de consejos estatutarios, la reducción de los sueldos de los funcionarios y la creación de un Centro de Estudios Políticos para moldear las actitudes de los funcionarios públicos (QuaH, 1975: 345-346). Se creó también una Oficina Central de Reclamaciones para controlar comportamientos inadecuados de los funcionarios públicos con los ciudadanos. La Ordenanza de Procedimiento Financiero de 1960 obligaba a los funcionarios públicos a rembolsar al gobierno cuando hubiese un manejo inadecuado de los fondos públicos (HIOK, 1980: 105). En 1972 se estableció el Consejo de Productividad Nacional para mejorar la productividad en todas las empresas en Singapur (TEO, 1985:315). En 1971 se estableció un Instituto de Formación de Personal para formar a nuevos funcionarios del Servicio Administrativo de la Función Pública de Singapur en conceptos y técnicas modemos de gestión (KHAN, 1998: 404). Para 1974 la formación alcanzaba al personal de otros servicios públicos y su alcance llegaba a incluir destrezas relacionadas con el lenguaje y la supervisión. Entre 1975 y 1979 se modificó dos veces el Instituto de Formación de Personal. En 1979 se transformó en Instituto de la Función Pública. La Oficina de Investigación de 
Prácticas Corruptas, ubicada en la Oficina del Primer Ministro, se fundó en 1952. La génesis de dicha Oficina parte de la Ley de Prevención de la Corrupción que se aprobó en 1937 y en la creación de la Oficina de Investigación de Prácticas Corruptas. El director de esta Oficina responde directamente ante el Primer Ministro. Las atribuciones y funciones de la misma han aumentado en las últimas dos décadas. Sus amplias atribuciones y responsabilidades incluyen: salvaguardar la integridad de la función pública, fomentar las transacciones libres de irregularidades en el sector privado, responsabilizarse del control e investigación de errores en la actuación de los funcionarios públicos, revisar los métodos de trabajo y procedimientos de los departamentos y organismos donde haya tendencia a las prácticas corruptas e identificar los puntos débiles en el procedimiento administrativo que puedan facilitar la corrupción y recomendar medidas preventivas y de solución (SINGAPORE, 1976: 53-54).

\section{Desarrollos recientes en reformas administrativas}

\section{Hong Kong}

En Hong Kong los desarrollos significativos en reforma administrativa empezaron con la publicación, en febrero de 1989, de un informe del gobierno titulado "Reforma del Sector Públi$\mathrm{CO}^{\circ}$, que contenía un plan del gobierno para una restructuración administrativa. El informe exigia reformas estructurales dentro del gobierno. Las recomendaciones del informe incluian: ensamblar más servicios públicos y corporaciones públicas después de su racionalización, contratar con el sector privado, en mayor escala, promover supresiones donde fueran apropiadas, establecer departamentos de comercio autocontrolados y desarrollo de un Sistema de Presupuestación basado en las Prioridades (Lo y Yuen, 1994: 3; Tang, Perry y LaM, 1994: 37). Otras iniciativas de reformas incluian: la racionalización de organismos no departamentales, la transmisión de responsabilidades sobre la gestión de los recursos, mayor énfasis en las funciones de política de gestión de las agencias de política central, cambios estructurales y de procedimiento dentro de la función pública para promover una toma de conciencia sobre los costes y resultados, y transformación de los funcionarios públicos de administradores a mejores gestores (CHEUNG, 1996: 31).

En octubre de 1992 el gobernador de Hong Kong, Chris Patten, introdujo un número de iniciativas bajo la rúbrica de servicio a la comunidad. Incluían: publicar la puesta en marcha de compromisos de cambio por el gobierno (SARII, 1995: 99). Estos cambios han sido posibles, de acuerdo con él, como resultado de la adopción de determinadas estrategias. Estas estrategias incluían: mejoras en la estructura, sistemas, normas y regulaciones y tecnología de la información, inculcando el valor de la calidad, productividad y responsabilidad en la función pública (SARII, 1995: 99).

Desde el principio de los años 80, el gobierno de Malasia se centró especialmente en una mejor gestión de los recursos para compensar las recesiones económicas. A este respecto se iniciaron un número de medidas, como disminución del servicio público a través de la privatización, la introducción del concepto de $\mathrm{Ma}$ laysian Incorporated y la inculcación de valores positivos y ética del trabajo (COMMONWEALTH SECRETARIAT, 1995 a: 1).

Las mayores reformas administrativas en la función pública de Malasia se pueden catalogar bajo cinco apartados: mantener la calidad del servicio, mejorar la productividad, asegurar la integridad y la responsabilidad e institucionalizar el éxito.

En relación con la calidad ya han sido implementadas bastantes medidas. Éstas incluyen Gestión Total de la Calidad, Red de Servicio Público y Conexión de la Función Pública, servicios de mostrador y carta de cliente. La Gestión Total de la Calidad se adoptó como un enfoque para movilizar todos los recursos disponibles en las agencias del sector público para satisfacer los requerimientos del cliente (SARII, 1995: 100). La Gestión Total de la Calidad se diseñó para crear más organizaciones centradas en el consumidor que sean viables y estén dispuestas a proporcionar mejores servicios a los clientes. Los departamentos gubernamentales fueron instruidos para formar Círculos de Control de Calidad. El objetivo de estos Círculos es promover las técnicas, experiencia y creatividad de los empleados en la resolución de problemas (COMmONWEalTH SECRETARIAT, 1995a: 3). La Red de Servicio Público y la Conexión de la Función Pública son también medidas para proporcionar información y servicios por medios electrónicos y para facilitar el comercio electrónico. Los permisos de circulación y de negocios se renuevan a traves de la Red de Servicio Público, utilizando las redes e instalaciones de ordenadores en las oficinas de correos. La Conexión de la Función Pública, por otro lado, actúa como un centro de información y abastece las necesidades del sector privado en áreas tales como permisos, licencias y tipos de impuestos. El objetivo del gobierno de Malasia es alejarse gradualmente de una era de burocracia de papeles (COMMONWEALTH SECRETARIAT, 1995b: 65). LOS Servicios de mostrador que tienen relación con la Red de Servicio Público y con la Conexión de la Función Pública, son otro dispositivo para proporcionar determinados servicios al público, como expedición de licencias, permisos, pasaportes, tarjetas de identidad, venta de sellos y cobro de facturas. El objetivo es proporcionar un servicio rápido, justo y cortés, de una forma regular, a los clientes. La Carta de cliente fue introducida en 1993. La Carta de cliente de una agencia del sector público in- 
cluye un compromiso escrito hecho por la agencia a sus usuarios: es una garantía de servicio (CHIU, 199: 176). El objetivo es proporcionar una completa satisfacción al cliente. Tanto la Carta de cliente como la Carta de ciudadano en el Reino Unido permiten que el cliente evalúe el servicio prestado de acuerdo con los estándares de calidad declarados y también permite a las agencias mejorar sus servicios de forma regular. Un componente clave de la Carta es el Sistema de Recuperación del Servicio que está diseñado para mejorar las deficiencias de la prestación de servicios, con miras a recobrar la confianza de los clientes (CHIU, 1997: 197).

Ya se han implementado determinadas medidas de política para mejorar la productividad. Éstas incluyen: un Nuevo Sistema de Remuneración, un Nuevo Sistema de Evaluación del Funcionamiento, la Política de Malaysian Incorporated, la privatización de las agencias del gobierno y la utilización de tecnología de la información. El Nuevo Sistema de Remuneración se puso en marcha el 1 de enero de 1992, y, a través, de la reclasificación, redujo la clasificación de los servicios de manera significativa, pasando de 574 a 19. Las 19 clasificaciones de los servicios estaban divididas en tres grupos: alta gestión, gestión y grupos profesionales y de apoyo. Significativamente, bajo el Nuevo Sistema de Remuneración, la promoción de un funcionario público, los incrementos del salario, la formación y colocación dependen de su comportamiento en el trabajo y su contribución hacia la organización y la función pública (COMMONWEALTH SECRETARIAT, 1995: 42). El Nuevo Sistema de Evaluación del Funcionamiento evalúa la actuación de un funcionario público de acuerdo con unos objetivos y metas establecidos previamente. La estrecha supervisión de la actividad se lleva a cabo de una manera rutinaria. La Politica de Malaysian Incorporated está basada en la filosofía que acerca la cooperación, colaboración y acción conjunta entre el gobierno y la industria (COMMONWEALTH SECRETARIAT, 1995:a: 4). Esto se considera esencial para un rápido desarrollo industrial. Consultores en los niveles federal, estatal y local son el vehículo para establecer diálogos anuales con el sector privado. Estos diálogos pretenden intercambiar información y opinión acerca de las actuaciones de las agencias del gobierno y de cómo afectan al sector privado. Como resultado de ello, los sistemas, normas y regulaciones de las agencias pueden cambiar para facilitar una creciente participación del sector privado en la economía. La privatización se utiliza para reducir el tamaño del sector público. Se ha establecido un Comité de Dirección para Reducir el Tamaño del Servicio Público. El Comité ha introducido determinadas medidas para alcanzar su objetivo de adelgazar el sector público. Las medidas incluyeron: control en la creación de puestos de trabajo, revisión del estado de los puestos vacantes, reorganización de las agencias bajo un Nuevo Sistema de Remuneración, revisión de la categoría de los organismos estatutarios, potenciación del ritmo del programa de privatización y uso de ordenadores y equipos de automatización de oficina (COMMONWEALTH SECRETARIAT, 1995:46). El gobierno proporciona, como una política, un fuerte apoyo al desarrollo de la tecnología de la información en el servicio público. Malasia gastó en 1993 243.000.000 \$M en tecnología de la información comparado con 197.000.000 \$M en 1992 (COMMONWEALTH SECRETARIAT, 1995b: 62). El gobierno ha emprendido un número importante de proyectos de tecnología de la información. Las agencias del gobierno han intensificado también la automatización de las oficinas para abordar con éxito la creciente carga de trabajo y los cambios en los requerimientos del trabajo.

Para asegurar la integridad y la responsabilidad en la función pública se han establecido determinados mecanismos y organismos. Los mecanismos de responsabilidad están incorporados en las leyes, regulaciones e instituciones que controlan el gasto público (COMmONWEalth SECRETARIAT, 1995a: 7). En 1982 la Ley de Auditoría fue enmendada convenientemente para incrementar el poder y responsabilidades del Auditor General a fin de que pudiera realizar una auditoría completa de las actividades de todas las agencias del gobierno a una escala más amplia Commonwealth SECReTariat, 1995a: 7). El Comité de Cuentas Públicas también juega un papel crucial para asegurar el control financiero por el Parlamento sobre la función pública. Este Comité también está ayudado por el informe del Auditor General presentado ante el Parlamento. La Oficina Pública de Reclamaciones, que está en el Departamento del Primer Ministro, recibe quejas directamente de los ciudadanos en caso de abuso de poder, interpretación errónea de las normas o mal comportamiento de los funcionarios públicos (CHIU, 1997: 179). El principal papel de la Oficina Pública de Reclamaciones es investigar las quejas de los ciudadanos contra los funcionarios públicos y las agencias y presentar los resultados a un poderoso Comité Permanente de Reclamaciones Públicas para una actuación apropiada. Una Agencia Anticorrupción hace cumplir estrictamente la Ley Anticorrupción de 1982. Dicha Agencia asegura que los funcionarios públicos que no estén envueltos en corrupción o comportamiento inadecuado sean recomendados para promoción y premios federales o estatales (COMMONWEALTH SECRETARIAT, 1995b: 48). El Sistema Modificado de Presupuestación es uno de los mecanismos para mejorar la gestión financiera y de responsabilidad en la función pública. Está orientado hacia medidas de resultados e impacto de los programas del gobierno, intensifica la responsabilidad de los funcionarios con responsabilidades de control en la gestión de los recursos que les han sido asignados. (COMMONWEALTH SECRETARIAT, 1995a: 8).

Se han utilizado tres estrategias para institucionalizar los éxitos conseguidos, hasta el momento, en el área de reforma administrativa en la función pública. Estas estrategias están destinadas a efectuar y mantener cambios importantes en los valores, conocimiento, destrezas y prácticas de los funcionarios 
públicos para llevar a cabo, de una manera efectiva, sus deberes de forma rutinaria. La publicación de Circulares de Desarrollo de la Administración, el establecimiento de un Sistema de Inspección y el énfasis en la formación y el perfeccionamiento en la gestión son algunas de las estrategias adoptadas para institucionalizar el éxito. Las Circulares de Desarrollo citadas contienen una guía detallada para implementar programas administrativos de mejora. El Sistema de Inspección se ha establecido para asegurar la puesta en marcha y el seguimiento de la implementación de los programas administrativos de mejora. El establecimiento y expansión del Instituto Nacional de Administración Pública como una organización de formación diversificada y con objetivos múltiples atestigua el énfasis continuado que pone el gobierno en la formación y el perfeccionamiento en la gestión y sus relaciones consiguientes con la institucionalización del éxito en la función pública.

\section{Singapur}

Singapur desde los primeros años de su independencia se movió de manera metódica y sistemática hacia la implementación de reformas administrativas. En los últimos años se han introducido e implementado medidas de reforma administrativa en aspectos clave en la función pública, con una frecuencia regular. Como resultado de ello, se han producido un número importante de cambios en toda la función pública. Las iniciativas de reforma más importantes incluían: devolución de la gestión de personal, autonomía a las agencias del gobierno, incremento de la productividad, institucionalización e integración del cambio, ampliación y potenciación de la calidad del servicio administrativo, utilización de tecnología de la información, freno de la corrupción, salarios competitivos para funcionarios destacados y reclutamiento selectivo de los mejores y más brillantes (KHAN, 1998:386-416; QUAH, 1995: 147).

A mediados de 1994 el gobierno instauró un equipo de altos funcionarios para supervisar los cambios en la gestión de personal en la función pública. Los Consejos de Personal de tres niveles fueron operativos desde el 1 de enero de 1995 como una iniciativa importante del gobierno para reformar la función pública (KHan, 1998: 389). En el nivel más bajo están los Consejos de Personal y en el más alto hay un Consejo de Personal Especial y entre ambos están los Consejos de Personal Superior. La mayor parte de los funcionarios públicos, es decir, los que pertenecen a las Divisiones II, III y IV están dirigidos por veinticuatro Consejos de Personal localizados en los Ministerios. Todo el personal de la División I está supervisado por seis Consejos de Personal Superior. Cada Consejo abarca tres o cuatro Ministerios. La jurisdicción del Consejo de Personal Especial incluye funcionarios de las escalas superiores hasta la escala E1 y el Personal del Servicio Administrativo en la escala temporal.
Los Ministerios han delegado el poder para contratar y promocionar a los funcionarios públicos a través del mecanismo de los Consejos de Personal. La intención es dotar de autoridad a los secretarios permanentes y a los gestores de línea para reclutar y promocionar a los candidatos que tengan más méritos. Los Consejos de Personal se guían en sus actividades por una serie de principios. Éstos son: mantener el mérito como base para la selección y promoción; un uso justo y consistente de la autoridad delegada para asegurar a todos el rigor y la equidad y proveer recursos de apelación a la Comisión de Servicio Público y otras Comisiones por parte de los funcionarios públicos agraviados. Como resultado de las últimas reformas, la Comisión de Servicio Público es ahora la responsable de la selección dentro del Servicio Administrativo, el Servicio Parlamentario y el Servicio de Auditoría; de la promoción del personal de la escala superior D y de los que están por encima; planificación y administración de las becas y bolsas de estudio para nacionales y para extranjeros, a nivel de graduado y licenciado, ofrecidas por el gobierno de Singapur; del despido y del control disciplinario y actuar como el Consejo de apelación final para oír las apelaciones contra las decisiones de promoción que tomen los consejos de personal. (SINGAPORE, 1997b: 52).

En marzo de 1996 el gobierno implementó una política para conceder mayor autonomía a los departamentos del gobierno, a las agencias y a los consejos estatutarios. En abril de 1997, excepto el Ministerio de Defensa y el Departamento de Seguridad Interna, todos los Ministerios y departamentos habían sido ya transformados en agencias autónomas. Estas agencias autónomas deben diseñar un plan de los servicios que pretenden ofrecer y reciben financiación, dependiendo de cómo cumplen una serie de criterios de actuación así como de la demanda que tienen sus servicios. El trabajo de las Agencias autónomas implica un número de pasos. Primero, cada agencia autónoma establece objetivos de actuación y de producción claramente definidos con los que también está de acuerdo, cada año, su Ministerio de origen y la División del Presupuesto del Ministerio de Finanzas. Segundo, el Ministerio de Finanzas proporcionará a las agencias autónomas un presupuesto sobre costes estimados para alcanzar los objetivos acordados. Pero la suma de dinero que recibirán las agencias depende de cómo cumplan sus objetivos y las demandas de servicio. Tercero, cada agencia autónoma es libre para gestionar el presupuesto de la forma que elija, siempre que cumpla sus objetivos. Las agencias disfrutan de una libertad considerable para decidir sobre la selección (por debajo del nivel de la escala superior) y sobre el despliegue de medios y tienen flexibilidad para utilizar los recursos económicos a su disposición, comparado con la situación en el pasado.

Acrecentar la productividad en el servicio público ha sido y continúa siendo un compromiso clave del gobierno. En 1981 se estableció un Consejo de Productividad Nacional para revisar y 
analizar los esfuerzos de productividad, para sugerir políticas y estrategias a efectos de mejorar la productividad entre la población (TEO, 1985: 317). En 1981 también comenzaron a funcionar un Comité Central de Dirección de la Productividad y el Movimiento de Productividad de la Función Pública. Como resultado de la decisión del Comité Central de Dirección de la Productividad, se introdujeron Equipos de Mejora del Trabajo en la función pública. Esto fue el anuncio del principio del Moviniento de Productividad de la Función Pública. El componente central de acrecentamiento de la productividad en la función pública gira alrededor de los Equipos de Mejora del Trabajo. Un Equipo de Mejora del Trabajo es un grupo de funcionarios públicos de la misma unidad de trabajo, independientemente de su status en el trabajo, que se reúnen regularmente para identificar, discutir y analizar problemas, encontrar soluciones a los mismos e implementar estas soluciones.

En el núcleo de la institucionalización y la integración del cambio está la iniciativa de Servicio Público para el siglo XXI. La iniciativa fue lanzada en mayo de 1995. Este Servicio incorpora otras iniciativas anteriores de mejora del sector público, como el Programa de Mejora de Contacto con el Público y la Unidad de Mejora del Servicio y los Equipos de Mejora del Trabajo. La Unidad de Mejora del Servicio, establecida en 1991 y ubicada en la Oficina del Primer Ministro, ha estado actuando como vigilante de los Ministerios y de los consejos estatutarios. Controla, audita y evalúa la calidad de los servicios que proporcionan las agencias del gobierno. El Servicio Público para el siglo XXI se centra en cuatro áreas: bienestar del personal, servicio de calidad, sugerencias de los Equipos de Mejora del Trabajo y del personal y revisión organizativa. La premisa de este Servicio es un enfoque comprensivo para preparar a los funcionarios públicos para que sean receptivos y capaces de cambiar utilizando su conocimiento apropiado (GUAN, 1997: 171). Se han desarrollado un número de iniciativas para funcionamiento de dicho Servicio. Se ha reunido y publicado un directorio de servicios públicos para que la gente sepa en qué departamento del gobierno puede hacer consultas para un servicio determinado. La introducción de un esquema de gratificaciones por atención al público que proporciona una gratificación mensual de $80 \$$ de Singapur a los funcionarios y empleados que trabajan detrás de los mostradores. Se está desarrollando un mapa de formación personal que contiene las necesidades de formación y los cursos que debe seguir un empleado. Algunas agencias del gobierno han establecido y publicado niveles de servicio que deben mantenerse en el trato con el público. Algunas agencias han establecido servicios de reclamaciones.

El Servicio Administrativo es el servicio élite entre todos los servicios públicos y disfruta de enorme poder. Se han implementado un número de medidas de reforma administrativa para ampliar la base y acrecentar la calidad del Servicio Administrati- vo. En 1990 se creó un Cuerpo de Administradores Superiores para incorporar a los mejores talentos al sector público. El esquema incluye, no sólo a los funcionarios del Servicio Administrativo y a los expertos de las Fuerzas Armadas de Singapur, sino también a los funcionarios de otros servicios. Otros cambios referentes a la gestión de personal del Servicio Administrativo incluyeron el establecimiento de unos criterios mínimos para el reclutamiento y la permanencia en el servicio. En 1995 se puso en marcha un Esquema de Carrera Dual. Bajo este esquema se seleccionaron veintiséis funcionarios de diferentes servicios para formar parte de los Servicios Administrativos. En 1996 los Servicios Administrativos abrieron sus puertas, en una escala muy limitada, al sector privado. La razón de esta actuación fue ayudar a la función pública para que estuviese permanentemente en sintonía con las tendencias exteriores y con las necesidades del sector privado. Existe también una actuación para destinar al personal del Servicio Administrativo a las compañias del sector privado, de forma que les permita ganar experiencia de primera mano sobre las condiciones de trabajo en el sector privado.

Con el establecimiento del Consejo Informático Nacional y la iniciación del Programa de Informatización de la Función Pública, ambos en 1981, comenzó la utilización sistemática de la tecnología de la información en la función pública. El Consejo se instauró para promocionar, implementar y dirigir el desarrollo de los sistemas de información en la función pública (QuaH, 1995: 151). El Programa de Informatización de la Función Pública intentaba facilitar un uso más amplio de los ordenadores por los funcionarios públicos para que de esa forma pudieran proporcionar un mejor servicio a la gente a través del uso de ordenadores (LENG, 1987: 6). Entre 1983 y 1985 se introdujo gran cantidad de recursos técnológicos para la telecomunicación y para el sistema de oficinas, tales como el Sistema de Red Telefónica de la Función Pública, los Servicios Telecom y Telebox y máquinas de fax, dando como resultado una reducción considerable en el coste de los servicios y una aceleración en el ritmo con que se proporcionaban estos servicios. En dos años, es decir, de 1997 a 1999, se van a introducir 8.000 nuevos ordenadores de sobremesa y 4.000 ordenadores personales para los funcionarios. El gobierno se estableció formalmente en Internet en 1996 como resultado de la disponibilidad de nuevos servicios en la Internet. Puede realizarse la solicitud de permiso de trabajo para las empleadas de hogar y el resultado puede obtenerse en tres días. La inscripción en las guarderías se ha facilitado con la posibilidad de acceso de los ciudadanos a bases de datos comprensivas de las guarderías reconocidas oficialmente. Está disponible una base electrónica del gobierno para posibilitar que la gente contacte con cualquier Ministerio o departamento por correo electrónico.

La corrupción en la función pública se ha reducido considerablemente debido a la adopción por el gobierno de una estra- 
tegia anticorrupción con dos componentes. En 1980 el gobierno enmendó la Ordenanza de Prevención de la Corrupción y la remplazó por la Ley de Prevención de la Corrupción para disminuir las oportunidades de comupción y para aumentar las penas por comportamiento comupto (QuaH, 1995: 148). La Oficina de Investigación de Prácticas Comuptas, ubicada en la Oficina del Primer Ministro y dotada de poderes adicionales por la ley, investiga y acusa a las personas dentro de los sectores público y privado para combatir eficazmente las prácticas corruptas. La mejora de los salarios y las condiciones de trabajo en la función pública también ha disminuido los incentivos para la corrupción.

Para retener a los mejores individuos se ofrecen salarios competitivos y condiciones de trabajo favorables a los funcionarios públicos. Ya en 1972 se otorgó a todos los funcionarios públicos una gratificación correspondiente a trece mensualidades comparable a las gratificaciones en el sector privado. Los salarios de los funcionarios superiores se aumentaron en 1973 para reducir la diferencia con sus homólogos en el sector privado, tendencia que continúa. En 1982 se revisaron los salarios de los miembros del Servicio Administrativo y de otros servicios profesionales para equilibrar las amplias disparidades que existían en los sueldos entre licenciados destacados en el sector privado y sus homólogos en la función pública, especialmente en el Servicio Administrativo. Después de la revisión más reciente, el salario para un funcionario del Servicio Administrativo, que trabaja suficientemente bien como para alcanzar la escala superior Grado $G$ a la edad de 32 años es de $12.400 \$$ al mes. Para atraer y retener personas de alto prestigio en el Servicio Administrativo su salario se vincula al salario medio de lo que gana el $15 \%$ superior en seis profesiones. Añádase a esto que la gratificación de los funcionarios del Servicio Administrativo ha variado de dos a tres en años recientes; una gratificación especial correspondiente a un mes de paga se da a un funcionario que lo merezca, una gratificación dependiendo de su actuación, que puede llegar hasta cuatro meses, se da a un funcionario de la escala superior $G$.

El gobierno sigue de cerca, constantemente, el reclutamiento selectivo de los mejores y más brillantes. La Comisión de Servicio Público ofrece becas atractivas a estudiantes brillantes no licenciados para estudiar en Singapur y en el extranjero. Alli, los expertos están obligados a servir en la función pública por un período fijo de tiempo. La Comisión también organiza charlas con estudiantes a punto de graduarse para conseguir candidatos cualificados en el mercado abierto. Asimismo, recluta candiditos para el Servicio Administrativo a través de entrevistas con candidatos previamente seleccionados.

\section{Conclusión}

Los tres países del Sureste asiático han tenido éxito en la iniciación e implementación de reformas administrativas de gran calado, consiguiendo cambios significativos en el tamaño, productividad y ética de trabajo en sus administraciones. Se mejoró la calidad de los servicios en términos de eficacia y efectividad. Se han desarrollado y puesto en práctica medidas de reforma proactiva apropiadas para enfrentarse a los cambios en el entorno y beneficiarse de ellos.

Las medidas de largo alcance de reforma administrativa han tenido éxito en Hong Kong, Malasia y Singapur debido a un número de razones. La comprensión de estas medidas puede proporcionar orientaciones importantes a otros países, desarrollados y en vías de desarrollo, en términos de estrategias y variables cruciales para la implementación de medidas importantes de reforma administrativa. Los líderes políticos, especialmente los de alto nivel en los tres países, no solamente proporcionaron orientación sino también un apoyo fuerte y sostenido a los csfuerzos de reforma. El compromiso político resultó ser la variable más importante para llevar a cabo las reformas. Los funcionarios superiores que mantenían posiciones claves en el gobierno proporcionaron en la mayoría de los casos un apoyo estusiasta a las medidas de reforma. También se colocaron funcionarios pro-reforma en posiciones claves para llevar a cabo las reformas, en algunas ocasiones, a través de terrenos difíciles y tortuosos. Tanto los máximos líderes políicos como los funcionarios superiores estuvieron dispuestos a tomar decisiones difíciles y emprender caminos de futuro incierto. Las reformas tuvieron éxito también porque se centraron en un número de aspectos claves que incluían: establecer metas claves, implicar tanto como fuese posible a las personas afectadas, examinar los procesos relevantes, medir el rendimiento, usar la tecnología y poner énfasis en los resultados. 
AltMAl), M.B. (1998) .Public Sector Governance and Administrative Reforms in Malaysia, documento presentado en la Mesa Redonda conjunta DESA/ECA sobre Goternance in Africa, Addis Ababa, Ethiopia, marzo 2-3.

Calden, G.E. (1991) Administrative Reforms Comes of Age. New York: Walter de Cruyter.

Cill:UNG, A.B.L. (1996) "Efficiency as the Rhetoric: Public-Sector Reform in Hong Kong Explained", Imtemational Revien of Administrative Sciences 62(1): 31-47.

Cillu, N.K. (1997) Service Targets and Methods of Redress: The Impact of Accountability in Malaysia, Public Adininistration and Detelopment (PAED) 17(1): 175-180.

Commonitinlm Stckimariat (1995a) A profile of the Public Sentice in Malaysia. London: Commonnealth Secretariat.

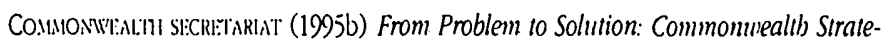
gies for Refonm. London: Commonwealth Secretariat.

GUAN, L.S. (1997) Sustaining Excellence in Government: The Singapore Experience, PAED 17(1): 167-174.

HıK, L.B. (1980) ·Personnel Relations in the Public Services of Singapore, en Rakasasataya, A. y Siedentoph, H. (eds.) Asian Sivil Senices: Technical Papers. Volume 8. Kuala Lumpur: Asian and Pacific Development Administration Centre, pp. 91-145.

HuQu!:, A.S. (1996) •Administering the Dragons: Clallenges and Issues en Huque., A.S. et al. (eds.) Public Administration in the NICs: Cballenges and Accomplisbments. New York: St. Martin's Press, pp. 1.32.

KIIAN, M.M. (1998) Reforms in the Singapore Civil Service: Lessons for Bangladesh., BlISS Joumal 19(4): 377-428.

LENG, L.S. (1987) -Productiviry Challenges for the Asian Senvices in the 21st CenturySingapore Experience incluido en ta Proceedings of the 4th. ASEAN Conference on Reforms in the Cinil Senvice: Productivity Challenges for the ASEAN Cinil Senvice in the 21si Century celebrada en Singapur, 27-31 de julio, pp. 1-15.

Lo, S.S.H. (1998) -The Changing Dimensions of Execulive - Legislative Relations: The Case of Hong Kong. Public Administration an Policy 7(2): 73-104.
Lo, C.W.H. y Peter, P.Y. (1994): ·Hong Kong Public Administration in Transition: An Overvien', Hong Kong Public Administration (HKPA) 3(1): 1-8.

QUnH, J.S.T. (1975) •Administrative Reform and Development Administration in Singapore: A Comparative Study of Singapore Improvement Trust and the Housing and Development Board. Unpublished Ph.D. Dissertation. Florida State University.

QUini, I.ST.T. (1995) S Sustaining Quality in the Singapore Civil Service. en Commonwealth Association for Public Administration and Management, (CAPAM), Goremment in Transition. London: Commonrealth Secretariat, pp. 147-157.

RAIMAN, A.B.A. (n.d.) -The Political and Administrative Context to the Reforms nithin the Malaysian Government Service aimed at Developing Quality Management. Unpublished paper.

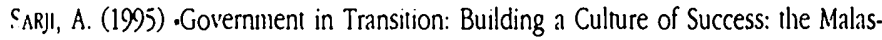
jan Experience. en CAPAM, Govermment in Transition. London: Commonwealth Secretarial, pp. 93-103.

SingaPore (1997a) Facts an Pictures 1997. Singapore: Ministry of Information and the Arts.

Singuporl: (1997b) Singapore 1997. Singapore: Ministry of Information and the Arts.

TANG, S.Y. ef al. (1994) . Changing Instiutional Contexts and Administmative Reform in Hong Kong. HKPA $3(1)$ : 31-44.

Tr:O, H.H. (1985) Singapore Civil Service: Genral Trends in the Civil Senvice Reforms en Trends in Civil Sentice Reforms in ASEAN Comntries. Bankok: Office of the Civil Service Commission, Royal Government of Thailand, pp. 311-332.

Vl:Ri, J.F.D. (1997) -Re-Engineering in the United Nations: A Reform in Process. Documento presentado en EROPA Conference on Public Service Mangement: Achieving Quality Performance in the 21st Century celebrada en Kuala Lumpur, Malysia del 1721 de noviembre, pp 1-15.

World Bank, The (1991) World Derelopment Report, 1991: The Challenge of Development. Oxford: Oxford University Press. 